\title{
The Inhibitory Effect of Hsa-miR-330 Replacement on the Proliferation and Migration of Breast Cancer MCF-7 Cells
}

\author{
Elham Hosseinzadeh $^{1,2}{ }^{\circledR}$, Behzad Mansoori $^{1,3}$, Ali Mohammadi ${ }^{1}$, Vahid Khaze ${ }^{1}$, Maryam Rezazadeh $^{2^{*}(\mathbb{D})}$ \\ Behzad Baradaran $^{1 *}$ (D)
}

\begin{abstract}
Objectives: miRNAs comprise a group of master gene expression regulators, exerting their effects after transcription through targeting specific mRNAs, hence, influencing cellular processes. A considerable number of miRNAs are known to affect cell proliferation and migration in breast cancer, one of which is hsa-miR-330, a key player in various types of cancers. The purpose of this study was to evaluate the anti-proliferative and anti-migrative effects of hsa-miR-330 on MCF-7 cell line.

Materials and Methods: MCF-7 cells were transfected with pCMV-miR-330 vector and cell selection was performed in media containing Geneticin (G418). Subsequently, MTT assay was performed to evaluate the effect of hsa-miR-330 on proliferation and scratch wound healing assay was employed to evaluate cellular migration. Finally, using real-time PCR, the expression of hsamiR-330 as well as the repressive impact on the expression of E2F was investigated.

Results: Upon confirmation of hsa-miR-330 induction in MCF-7 cells via GFP channel imaging system, miR-330 expression was demonstrated to be increased 10 folds in stable cells. The results of MTT and wound-healing assays demonstrated an inhibitory role for hsa-miR-330 in proliferation and migration of stable hsa-miR-330-transfected MCF-7 cancer cells compared to controls. In addition, after transfecting cells with hsa-miR-330, E2F1 was down-regulated in comparison with controls.

Conclusions: Based on the results of the current study, we suggest a potential inhibitory role for hsa-miRNA-330 in cell proliferation as well as cell migration in breast cancer by targeting E2F1 mRNA. Additionally, a therapeutic role can be suggested for hsa-miR-330 in terms of target therapy for breast cancer.

Keywords: Breast cancer, E2F1, Hsa-miR-330, Cancer therapy
\end{abstract}

\section{Introduction}

According to the National Institutes of Health (NIH) reports, breast cancer ranked first in prevalence among females (1). During invasion, breast cancer cells manage to permeate out of lobules or ducts, migrate towards closely-located tissues, and thus contribute to a higher risk of metastasis (2). The treatment efficacy in breast cancer depends on the type, stage, sensitivity to hormones, and the age of the affected person. Today, the strategies for breast cancer treatment include radiation therapy, surgery, hormone therapy, chemotherapy, and biological therapy, or targeted drug therapy (3).

Recent studies on breast cancer focus on finding different mechanisms underlying the pathogenesis of this cancer. Understanding the biological and molecular mechanisms involved in breast carcinogenesis helps scientists to utilize the novel strategies in targeted therapy for breast cancer. Recently, miRNAs have been introduced as a master regulatory molecule in different cellular processes, including growth, differentiation, apoptosis, and so on $(4,5)$. These mechanisms draw the attention of researchers to utilize miRNAs as a novel molecular pathway in targeted therapies for breast cancer.

miRNAs comprise a group of small non-coding RNAs that suppress the expression of target genes after transcription has taken place. Studies have suggested that levels of miRNA expression are dysregulated in most types of cancer $(6,7)$. Restoring the transcription of tumor suppressor miRNAs to physiological levels is known as miRNA replacement therapy (8). Piva et al discussed the advantage of miRNA replacement therapy as novel clinical tools for breast cancer in their literature review (9). Several miRNAs including miR-7 (10), miR-30a (11), and miR-125b (12) were evaluated in breast cancer cells using miRNA replacement strategy and the results showed that these small molecules could be potentially effective in treating breast cancer.

Hsa-miR-330 gene, discovered in 2005 by Weber, is located on chromosome 19 in human and 7 in mouse (13). Experiments have demonstrated that hsa-miR-330 can regulate apoptosis and cellular proliferation $(14,15)$. In a recent study on hsa-miR-330 in a colorectal cell line,

Received 12 January 2018, Accepted 26 March 2018, Available online 3 May 2018

${ }^{1}$ Immunology Research Center, Tabriz University of Medical Sciences, Tabriz, Iran. ${ }^{2}$ Department of Medical Genetics, Faculty of Medicine, Tabriz University of Medical Sciences, Tabriz, Iran. ${ }^{3}$ Student Research Committee, Tabriz University of Medical Sciences, Tabriz, Iran.

*Corresponding Authors: Maryam Rezazadeh, Email: rezazadeh.mary@gmail.com; Behzad Baradaran, Tel: +984133371440, Fax: 984133371311, Email: Behzad_im@yahoo.com 
it was observed that the induction of the aforementioned miRNA halts the cell cycle at G1 phase, leading to the inhibition of cell proliferation (14). In another study, Lee et al introduced hsa-miR-330 as tumor suppressor miRNA, elucidating miR-330 role in the inhibition of prostate tumor proliferation by suppressing the phosphorylation of protein kinase B (AKT) (16).

Although a large number of miRNAs have been recognized as significant performers in breast cancer mechanobiology, the role of hsa-miR-330 is not clearly known in breast cancer. In this study, we evaluated the role of hsa-miR-330 in cell proliferation as well as cell migration in breast cancer, along with the investigation of the role of hsa-miR-330 in the regulation of $\mathrm{E} 2 \mathrm{~F} 1$ expression.

\section{Materials and Methods}

Bacterial Culture and Transformation Procedure

Escherichia coli DH5-Alpha was used for vector amplification. To accept the pCMV-miR-330 plasmid (Origene, USA) by bacteria, the competence of the bacteria was evaluated prior to transformation (Figure 1). To enhance the permeability of the bacterial membrane, 100 $\mathrm{mM}$ of glycerol and $\mathrm{CaCl} 2$ were used at low temperature on ice. PCMV-miR-330 plasmid was added to microtubes containing bacteria. The microtubes were then mixed and incubated for 30 minutes at $4^{\circ} \mathrm{C}$. Then, the microtubes were placed in a $42^{\circ} \mathrm{C}$ water bath for 60 seconds to further increase the gaps on the bacterial cell wall, permitting the pCMV-miR-330 plasmid to enter into the bacteria. Afterwards, the microtubes were kept on ice and LuriaBertani (LB) broth was added and then, the microtubes were incubated at $37^{\circ} \mathrm{C}$ in a shaker incubator at $170 \mathrm{rpm}$ for 1 hour. In order to reach sedimentation, the microtubes were centrifuged for 5 minutes at $2400 \mathrm{rpm}$. The bacterial supernatant was cultured on LB agar medium containing kanamycin and incubated at $37^{\circ} \mathrm{C}$. After the growth of vector-positive bacteria, the transformed bacteria were transferred to LB broth medium. Extraction of plasmid

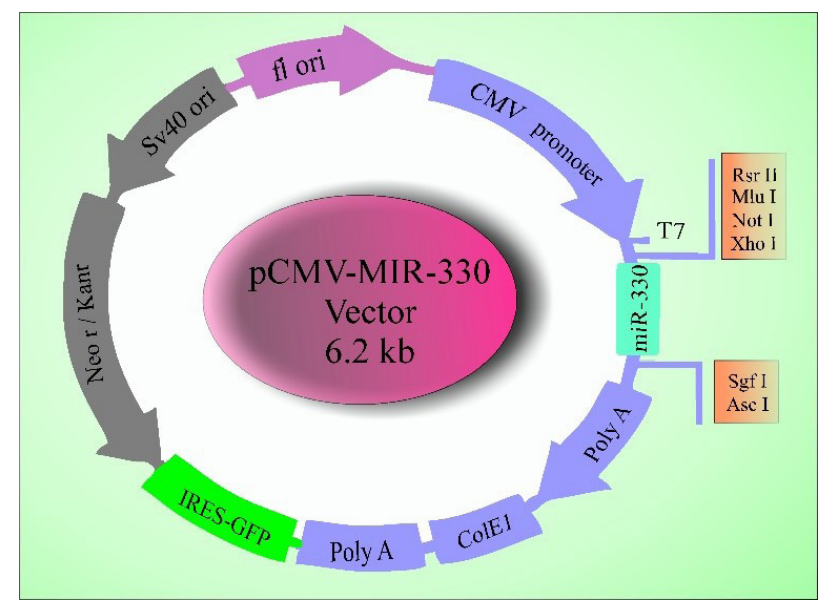

Figure 1. Schematic Drawing of PCMV-MIR-330 Construct.
DNA was performed using YTA Plasmid DNA Extraction Mini and Maxiprep kit (YektaTajhiz, Iran). Using NanoDrop (Thermo Scientific, 2000c, USA), the optical density (OD) of extracted plasmid was measured and DNA was then stored at $-20^{\circ} \mathrm{C}$.

\section{MCF-7 Cell Transfection With Hsa-miR-330}

The breast cancer cell line MCF-7 was purchased from the cell bank (Pasteur Institute of Iran), cultured in the RPMI 1640 medium (Gibco, USA), enriched with $10 \%$ fetal bovine serum (FBS) (Gibco, USA), and incubated at $37^{\circ} \mathrm{C}$ in an atmosphere of $95 \%$ humidity and $5 \% \mathrm{CO}_{2}$. Transfection was conducted using jetPEI ${ }^{\circledR}$ solution (Polyplus, France) according to the manufacturer's protocol. A total of $1 \times 10^{6}$ cells were seeded in 6-well plates and the transfection was performed in antibiotic and FBS free conditions according to manufacturer's manuals. Briefly, $6 \mu \mathrm{L}$ of the jetPEI solution and $188 \mu \mathrm{L}$ of $150 \mathrm{mM} \mathrm{NaCl}$ solution (Polyplus, France) were added to a microtube and $6000 \mu \mathrm{g} / \mathrm{mL}$ of the pCMV-miR-330 construct (Origene, USA) was added to the same microtube. Then, the microtubes were mixed gently and incubated for 30 minutes at room temperature. Next, the mixture containing JetPEI and pCMV-miR-330 was added to the cells in the plates and was then centrifuged at $200 \mathrm{~g}$ for 5 minutes. This method was also performed for an empty vector without any miRNA gene (control group). Afterwards, $100 \mu \mathrm{L}$ of FBS was added to each well after 6 hours and incubated for 24 hours. Plates containing cells were checked after 24 hours and the antibiotic-free medium was replaced with culture medium (RPMI1640) containing 20\% FBS and antibiotics.

Since transfected pCMV construct contains G418/ neomycin-resistance gene, in order to select pCMVmiR-330 construct positive cells, $8 \mu \mathrm{L}(50 \mathrm{mg} / \mathrm{mL})$ of G418 (Geneticin, Gibco, USA) was added to each well of the plate. To verify the successful transfection of cells with pCMV-miR-330 construct, wells were checked with GFP channel of Cytation 5 device (Biotek, USA). Each well was analyzed separately by bright field and GFP channels and the images were analyzed via Gene $5^{\mathrm{TM}}$ software (Biotek, USA, Version 1.1) (8).

\section{Quantitative Real-Time PCR}

The whole RNA content of stable hsa-miR-330 transfected and control MCF-7 cells was extracted using the Trizol method. Briefly, the pellet of cells was collected from the microtubes. Total RNA was extracted using RiboEx reagent (GeneAll biotechnology, Korea) following the manufacturer's instructions. The concentration of extracted total RNA was evaluated by NanoDrop device (Thermo Scientific, 2000c, USA).

In order to synthesize complementary DNA (cDNA) for evaluating the expression level of hsa-miR-330, cDNA Synthesis kit (Exiqon, Denmark) was used. To synthesize cDNA, $10 \mathrm{ng} / \mu \mathrm{L}$ of total RNA, $1 \mu \mathrm{L}$ of enzyme mix, and $2 \mu \mathrm{L}$ of $5 \mathrm{x}$ reaction buffer were mixed in microtubes. 
The mixture was incubated for 60 minutes at $42^{\circ} \mathrm{C}$ and 5 minutes at $95^{\circ} \mathrm{C}$ in reverse transcription PCR device (BioRad, T100 Thermal Cycle, USA).

Hsa-miR-330 expression was measured using SYBR Green PCR master mix and Light Cycler 96 instrument (Roche Diagnostics, Mannheim, Germany). According to the manufacturer's protocol, the cDNA was diluted 1:80, and then, $4 \mu \mathrm{L}$ of diluted $\mathrm{cDNA}$ was added to real-time PCR strips. Afterwards, $5 \mu \mathrm{L}$ of the SYBR Green master mix (EXIQON, Denmark) and $1 \mu \mathrm{L}$ of primers (EXIQON, Denmark) for hsa-miRNA-330 (Table 1) were added to each strip. The strips were loaded into Light Cycle 96 instrument. The real-time PCR temperature conditions were as follows: 60 seconds of enzyme activation at $95^{\circ} \mathrm{C}$, followed by 45 cycles of $95^{\circ} \mathrm{C}$ for 10 seconds, and $60^{\circ} \mathrm{C}$ for 60 seconds at a ramp rate of $1.6^{\circ} \mathrm{C} / \mathrm{s}$. The transcript level of SNORD48 was used as the housekeeping gene.

The cDNA Synthesis Kit (Thermo Scientific, USA) was used for cDNA synthesis of target gene (E2F1). The expression level of E2F1 mRNAs was measured by realtime PCR using SYBR Green PCR master mix and Light Cycler 96 instruments. Briefly, $0.5 \mu \mathrm{L}$ of cDNA, $5 \mu \mathrm{L}$ of SYBR Green master mix (Ampliqon, Denmark), and 0.25 $\mu \mathrm{L}$ ( 4 pmol) of forward and reverse primers (Oligo, Korea) of each gene were added to each of the strips. Primer sets are shown in Table 1 with more details. Finally, $\mathrm{H}_{2} \mathrm{O}$ was added to each strip to a final volume of $10 \mu \mathrm{L}$. The real-time PCR temperature conditions were as follows: 90 seconds of enzyme activation at $95^{\circ} \mathrm{C}$, followed by 35 cycles of $95^{\circ} \mathrm{C}$ for 10 seconds, $59^{\circ} \mathrm{C}$ for 35 seconds, and $72^{\circ} \mathrm{C}$ for 20 seconds. GAPDH expression level was used as the housekeeping gene. Finally, the relative levels of mRNA expression were measured by the comparative $\mathrm{C}_{\mathrm{T}}$ approach using $2^{-\Delta \mathrm{CT}}$ formula (17).

\section{MTT Cell Proliferation Assay}

A total of $2 \times 10^{3}$ cells of both hsa-miR330 transfected and control groups were cultured in triplicate in 96well plate for 48 hours. The medium of each well was replaced with a solution containing $50 \mu \mathrm{L}(2 \mathrm{mg} / \mathrm{mL})$ of MTT (Sigma-Aldrich, USA) and $100 \mu \mathrm{L}$ of RPMI 1640 medium containing 10\% FBS; then, the plates were incubated at $37^{\circ} \mathrm{C}$ for 4 hours in darkness. Subsequently, the supernatant was discarded and $200 \mu \mathrm{L}$ of DMSO was added to each well to be incubated for 30 minutes at $37^{\circ} \mathrm{C}$. Finally, the absorbance measurement was performed at $570 \mathrm{~nm}$ wavelength for each well using a microplate reader (Tecan, Sunrise, Austria) (18).
Wound Healing Assay

In order to determine if hsa-miR-330 is involved in cellular migration, wound healing assay was employed. Totally, $5 \times 10^{5}$ MCF-7 cells of both hsa-miR-330 transfected and control groups were cultured in 24-well plates with RPMI 1640 medium containing $10 \% \mathrm{FBS}$ to be incubated at $37^{\circ} \mathrm{C}$ for 24 hours. Afterwards, the cells were allowed to fill a monolayer plate; then a scratch was created by a yellow pipet tip in each well, at $90 \%$ confluency. Thereafter, the migration of cells into the scratch wound area in both hsamiR-330 transfected and control cells were analyzed by an inverted microscope (Optika, Italy) and compared at different time points $(0,24$, and 48 hours).

\section{Statistical Analysis}

Data analyses were entirely done by GraphPad Prism statistical software, version 7.0 (Graph Pad Software Inc., San Diego, CA, USA). All values were expressed as mean \pm standard deviation (SD). The Kolmogorov-Simonov test was performed to evaluate the normality of the variables. Student's $t$ test and two-way analysis of variance (ANOVA) were employed to determine the statistical differences between the groups. A $P$ value of less than 0.05 was considered statistically significant.

\section{Results \\ pCMV-miR-330 Construct Could Induce hsa-miR-330 Expression in MCF-7 Cells \\ For stable expression of hsa-miR-330 in MCF-7 cell line, pCMV-miR-330 vectors were transfected into the cells; then, the cells which received the vector were selected by treating with G418 (Figure 2A and 2B). It was observed that the expression of hsa-miR330 was increased 11.37 folds $(P<0.001)$ in transfected cells compared with the negative vector control group (Figure 2C).}

hsa-miR-330 Replacement Reduced E2F1 mRNA Expression in MCF-7 Cells

E2F1 mRNA in hsa-miR-330-transfected MCF-7 cells was down-regulated significantly $(P<0.01)$ compared with the control group (Figure 3). Therefore, E2F1 expression could be modulated by hsa-miRNA-330.

hsa-miR-330 Could Reduce Breast Cancer Cell Proliferation

According to Figure 4, the results of MTT assay showed that the percentage of cell growth inhibition $(10.43 \pm 3.79$ $\%)$ in hsa-miR-330-transfected cells was significantly lower $(P<0.0001)$ than that in control group $(89 \pm 3.8 \%)$.

Table 1. Primer Sequences

\begin{tabular}{lll}
\hline Gene & Sequence $\left(\mathbf{5}^{\prime} \mathbf{- 3} \mathbf{)}\right.$ & \\
\hline hsa-miR-330 & Target sequence & CUUUGGCGAUCACUGCCUCUCUGGGCCUGUGUCUUAGGCUCUGCAAGA \\
& & UCAACCGAGCAAAGCACACGGCCUGCAGAGAGGCAGCGCUCUGCCC \\
E2F1 & Forward & GACGTGTCAGGACCTTCGTA \\
& Reverse & GACCAAAACAGCGAGGAAGC \\
\hline
\end{tabular}



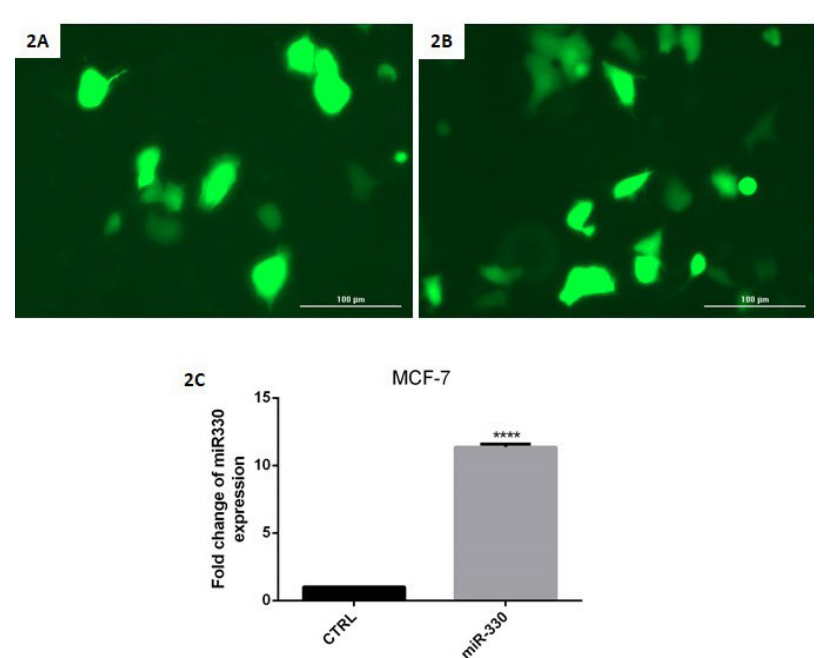

Figure 2. PCMV-miR-330 Construct Replaced into MCF-7 Cells. HsamiR-330 (2A) and miRNA empty vector (2B) expressions were confirmed by imaging with cytation 5 system in GFP channel. In addition, miR-330 level elevated in miR-330 stable cells compared to controls (2C). Data are presented as mean $\pm \mathrm{SD}(\mathrm{n}=3)$; ${ }^{* * *} P<0.001$ versus empty vectortransfected control cells (CTRL).

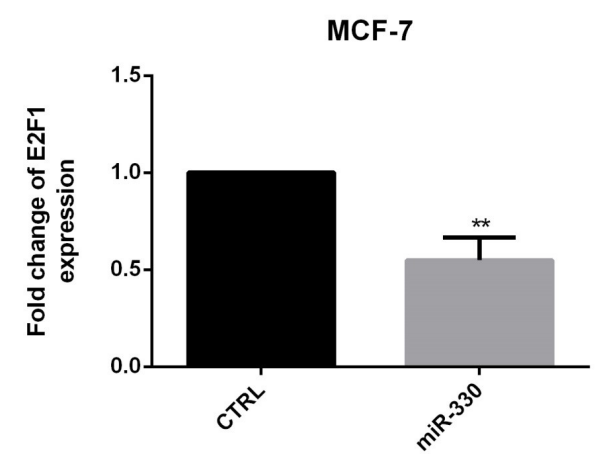

Figure 3. The Effect of miR-330 Induction on E2F1 mRNA in Breast Cancer MCF-7 Cell Line. E2F1 expression Levels decreased in miR-330 replaced cells (miR-330) compared with empty vector control (CTRL). Data are presented as mean $\pm \mathrm{SD} .{ }^{*} P<0.01$ versus empty vector control.

hsa-miR-330 Decreased MCF-7 Cell Migration

After transfection (the 0 time point), the number of migrated cells in hsa-miR-330 transfected cells and control MCF-7 cells did not show any significant difference. However, it was observed that the mean number of cells migrated to the wound area 24 hours after wounding was $75 \pm 4.5$ in hsa-miR-330 transfected cells, which was significantly lower $(P<0.01)$ compared to migrated cells in control MCF-7 cells (163 \pm 7$)$. Moreover, the mean number of migrated cells after 48 hours was $242 \pm 10.4$ in hsa-miR-330 transfected cells and it was $613 \pm 25.9$ in control cells, which was significantly different $(P<0.01$; Figure $5 \mathrm{~A}$ and $5 \mathrm{~B})$.

\section{Discussion}

miRNAs are prominent key regulatory molecules, which regulate biological process including proliferation, differentiation, apoptosis, migration and many

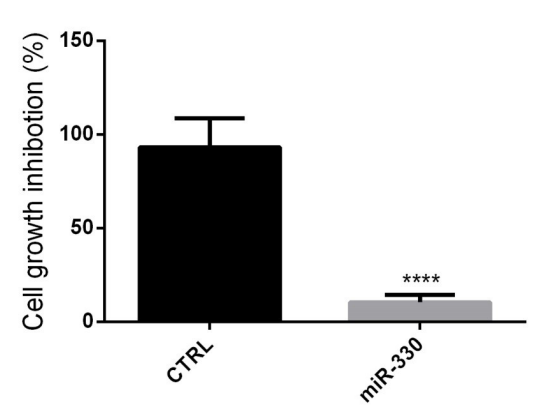

Figure 4. The Effect of miR-330 Replacement on the Viability of Breast Cancer Cells. The data are presented as mean \pm SD $(n=3) ;{ }^{* * *} P<0.01$ versus empty vector-transfected control cells (CTRL).

pathological processes, especially in cancer (19). Aberrant miRNA expression is closely linked to different human diseases like cancer (4). Moreover, numerous studies on breast cancer have been conducted to clarify their specific roles as well as their targets; however, the expression level, function, and target of some miRNAs in breast cancer remain unclear $(4,20)$. Previous cancer studies on hsa-miR-330 indicated that this miRNA could act as either tumor suppressor or oncogenic miRNA $(14,21)$. Meng et al suggested that hsa-miR-330 might function as an oncomiR in human esophageal cancer (22). On the contrary, Tréhoux et al reported miR-330-5p and miR-29a as tumor suppressor miRNAs in pancreatic cancer cells (23). Specific searching in Human miRNA Expression Database (miRmine, University of Michigan) demonstrated that the expression level of hsa-miR-330 was decreased in tumor tissues compared with the normal tissue from patients with breast cancer. These results confirm the role of hsa-miR-330 as a tumor suppressor in breast cancer.

The results of this study showed that hsa-miR-330 could inhibit the migration of cancer cells. Hsa-miR-330 was induced in MCF-7 cell line by transfection of GFP-pCMVmiRNA construct. In addition, the hsa-miR-330 induction was confirmed to be increased 10 folds compared with the empty miRNA vector group. According to the results of the wound healing assay, hsa-miR-330 could inhibit cell migration in breast cancer cell lines. Furthermore, the results indicated that hsa-miR-330 transfection could inhibit cell migration by about $44 \%$ in 24 hours; however, the inhibition in 48 hours was 39\%. These results suggest that miRNA replacement could exert an inhibitory effect on cell migration in a time-dependent manner.

The results of MTT assay revealed that hsa-miR-330 could significantly reduce breast cancer cell proliferation. More than $80 \%$ reduction in the growth of breast cancer cells implies that this miRNA has a beneficial role in inhibiting cancer cell proliferation. Similarly, Lee et al demonstrated that miR-330 serves against proliferation in prostate cancer cells (16). On the other hand, our results are in contrast to the study by Qu et al who observed hsamiR-330 overexpression in glioblastoma promoted cellular 

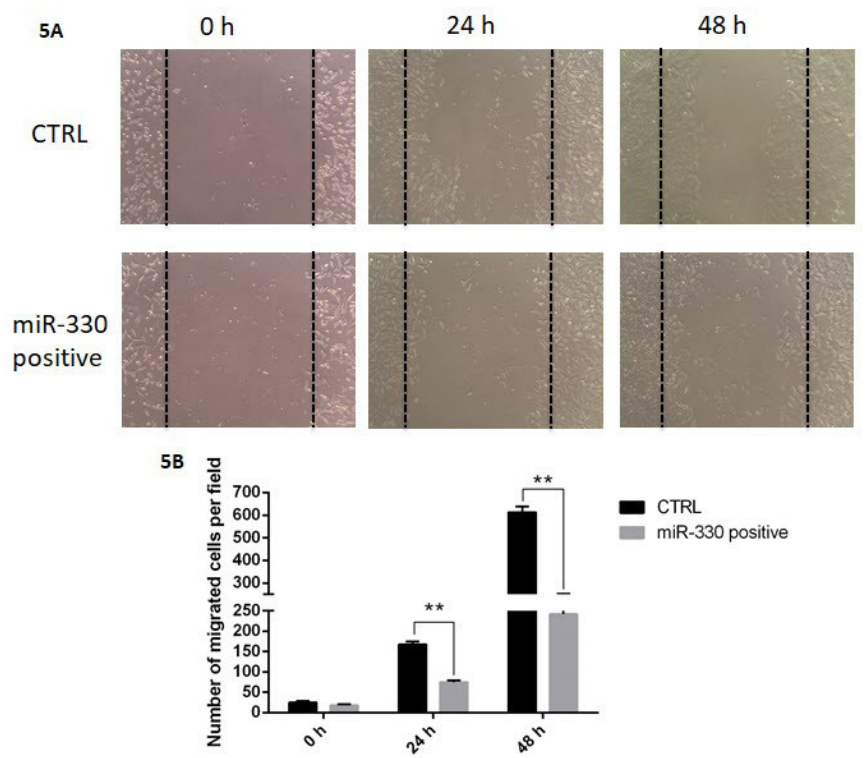

Figure 5. The Effect of PCMV-miR-330 Construct on Breast Cancer cell Migration. The stable hsa-miR-330 expressed cells were scratched and the scratch area was observed after 24 and 48 hours using inverted microscopy [as explained in the Methods section] (5A). The number of cells migrated into the scratch area was counted and the number of miR-330-induced cells was compared with that of the control group (empty pCMV vector) at 0,24 and 48 hours (5B). Data are presented as means $\pm S D(n=3) ;{ }^{* *} P<0.01$ versus empty vector controls.

proliferation, migration, and invasion by regulating SH3GL2 gene (known as oncomiR) (21). Moreover, Liu et al showed miR-330 targets early growth response 2 (EGR2), and could, therefore, control cell proliferation in non-small-cell lung cancer (24). It was also reported that miR-330 could modulate CTNNB1 expression and this cause the suppression of IL-22-induced keratinocyte proliferation (25). Furthermore, the suppression of migration has been detected in the miR-330 induced cells, but this might be the significant role of miRNA in cancer cells. The results of the current study are in line with the study by Kim et al, who demonstrated the potential inhibitory effect of miR-330-5p on the proliferation and migration of keratinocytes in mouse (26). Additionally, they showed that these effects might be directly mediated by miR-330-5p as a regulatory factor in Pdia 3 mRNA. The results are still inconclusive with respect to the exact function of hsa-miR-330 in inhibiting tumor progression.

The result of E2F1 gene expression showed that the relative expression of this gene was significantly decreased in miR-330 transfected cells compared with the control group. Moreover, hsa-miR-330 could decrease E2F1 level by targeting the corresponding mRNA. Studies confirmed that overexpression of E2F1 was linked to ontogenesis and neoplastic transformation (27). Previous studies showed the inhibition of cell proliferation and migration through silencing E2F1 gene by specific siRNA (28). E2F1, in turn, activates downstream factors and results in proliferation and migration of cancer cells (29). It was observed that E2F1 induced cancer cell proliferation by upregulating cyclin $\mathrm{E}$ and A2 (30). E2F1 also induced cancer cell migration by regulation of E-cadherin, $\mathrm{N}$-cadherin, vimentin, and snail $(31,32)$. It seems that abnormal expression of hsa-
miR-330 can participate in tumor pathology; hence, therapeutic approaches targeting this molecule could have positive effects, at least in breast cancer.

\section{Conclusions}

The conducted experiment suggests that hsa-miR-330 replacement exerts antiproliferative and anti-migrative effects on breast cancer cell lines, which possibly occurs through the inhibition of E2F1 expression. The results of the current study shine a light on the outstanding role of hsa-miR-330 and its target, E2F1, in breast cancer. Therefore, the results suggest that hsa-miR-330 has potential tumor-suppressive effects on breast cancer cells and might also be therapeutically useful in the treatment of patients with breast cancer in the near future.

\section{Conflict of Interests}

Authors declare that they have no conflict of interests.

\section{Ethical Issues}

All the methods of this study were approved by the Ethics Committee of Tabriz University of Medical Sciences with the reference code of IR.TBZMED.REC.1395.951.

\section{Financial Support}

This work was supported by the Vice Chancellor for Research of Tabriz University of Medical Sciences.

\section{References}

1. Mullooly M, Khodr ZG, Dallal CM, et al. Epidemiologic risk factors for in situ and invasive breast cancers among postmenopausal women in the National Institutes of Health-AARP diet and health study. Am J Epidemiol. 2017;186(12):1329-1340. doi:10.1093/aje/kwx206 
2. Mina LA, Storniolo AM, Kipfer HD, Hunter C, Ludwig KK. Breast cancer prevention and treatment. Springer; 2016:5-11. doi:10.1007/978-3-319-19437-0

3. Gordon A. The increasing efficacy of breast cancer treatment. Clin Oncol (R Coll Radiol). 1997;9(5):338-342. doi:10.1016/ S0936-6555(05)80069-X

4. Mansoori B, Mohammadi A, Shirjang S, Baradaran B. MicroRNAs: The new potential biomarkers in cancer diagnosis, prognosis and cancer therapy. Cell Mol Biol (Noisy-legrand). 2015;61(5):1-10.

5. Mansoori B, Mohammadi A, Shirjang S, Baradaran B. MicroRNAs in the diagnosis and treatment of cancer. Immunol Invest. 2017;46(8):880-897. doi:10.1080/0882013 9.2017.1377407

6. Shirafkan N, Mansoori B, Mohammadi A, Shomali N, Ghasbi M, Baradaran B. MicroRNAs as novel biomarkers for colorectal cancer: New outlooks. Biomed Pharmacother. 2018;97:1319-1330. doi:10.1016/j.biopha.2017.11.046

7. Mohammadi A, Mansoori B, Baradaran B. The role of microRNAs in colorectal cancer. Biomed Pharmacother. 2016;84:705-713. doi:10.1016/j.biopha.2016.09.099

8. Tavanafar F, Safaralizadeh R, Hosseinpour-Feizi MA, et al. Restoration of miR-143 expression could inhibit migration and growth of MDA-MB-468 cells through down-regulating the expression of invasion-related factors. Biomed Pharmacother. 2017;91:920-924. doi:10.1016/j. biopha.2017.04.119

9. Piva R, Spandidos DA, Gambari R. From microRNA functions to microRNA therapeutics: novel targets and novel drugs in breast cancer research and treatment (Review). Int J Oncol. 2013;43(4):985-994. doi:10.3892/ijo.2013.2059

10. Pogribny IP, Filkowski JN, Tryndyak VP, Golubov A, Shpyleva SI, Kovalchuk O. Alterations of microRNAs and their targets are associated with acquired resistance of MCF-7 breast cancer cells to cisplatin. Int J Cancer. 2010;127(8):1785-1794. doi:10.1002/ijc.25191

11. Zhang N, Wang X, Huo Q, et al. MicroRNA-30a suppresses breast tumor growth and metastasis by targeting metadherin. Oncogene. 2014;33(24):3119-3128.

12. Wang HJ, Guo YQ, Tan G, et al. miR-125b regulates side population in breast cancer and confers a chemoresistant phenotype. J Cell Biochem. 2013;114(10):2248-2257. Weber MJ. New human and mouse microRNA genes found by homology search. FEBS J. 2005;272(1):59-73. doi:10.1111/ j.1432-1033.2004.04389.x

13. Li Y, Zhu X, Xu W, Wang D, Yan J. miR-330 regulates the proliferation of colorectal cancer cells by targeting Cdc42. Biochem Biophys Res Commun. 2013;431(3):560-565. doi:10.1016/j.bbrc.2013.01.016

14. Yao Y, Xue Y, Ma J, et al. MiR-330-mediated regulation of SH3GL2 expression enhances malignant behaviors of glioblastoma stem cells by activating ERK and PI3K/ AKT signaling pathways. PLoS One. 2014;9(4):e95060. doi:10.1371/journal.pone.0095060

15. Lee KH, Chen YL, Yeh SD, et al. MicroRNA-330 acts as tumor suppressor and induces apoptosis of prostate cancer cells through E2F1-mediated suppression of Akt phosphorylation. Oncogene. 2009;28(38):3360-3370. Livak KJ, Schmittgen TD. Analysis of relative gene expression data using real-time quantitative PCR and the 2(-Delta Delta
C(T)) Method. Methods. 2001;25(4):402-408. doi:10.1006/ meth.2001.1262

16. Ahmadian S, Barar J, Saei AA, Abolghassemi Fakhree MA, Omidi Y. Cellular toxicity of nanogenomedicine in MCF-7 cell line: MTT assay. J Vis Exp. 2009(26). doi:10.3791/1191

17. Mohammadi A, Mansoori B, Baradaran B. Regulation of miRNAs by herbal medicine: An emerging field in cancer therapies. Biomed Pharmacother. 2017;86:262-270. doi:10.1016/j.biopha.2016.12.023

18. Mansoori B, Mohammadi A, Shirjang S, Baghbani E, Baradaran B. Micro RNA 34a and let-7a expression in human breast cancers is associated with apoptotic expression genes. Asian Pac J Cancer Prev. 2016;17(4):1887-1890. doi:10.7314/ APJCP.2016.17.4.1887

19. Qu S, Yao Y, Shang C, et al. MicroRNA-330 is an oncogenic factor in glioblastoma cells by regulating SH3GL2 gene. PLoS One. 2012;7(9):e46010. doi:10.1371/journal.pone.0046010

20. Meng H, Wang K, Chen X, et al. MicroRNA-330-3p functions as an oncogene in human esophageal cancer by targeting programmed cell death 4. Am J Cancer Res. 2015;5(3):10621075.

21. Trehoux S, Lahdaoui F, Delpu Y, et al. Micro-RNAs miR-29a and miR-330-5p function as tumor suppressors by targeting the MUC1 mucin in pancreatic cancer cells. Biochim Biophys Acta. 2015;1853(10 Pt A):2392-2403. doi:10.1016/j. bbamcr.2015.05.033

22. Liu X, Shi H, Liu B, Li J, Liu Y, Yu B. miR-330-3p controls cell proliferation by targeting early growth response 2 in nonsmall-cell lung cancer. Acta Biochim Biophys Sin (Shanghai). 2015;47(6):431-440. doi:10.1093/abbs/gmv032

23. Shen H, Zeng B, Wang C, et al. MiR-330 inhibits IL-22induced keratinocyte proliferation through targeting CTNNB1. Biomed Pharmacother. 2017;91:803-811. doi:10.1016/j.biopha.2017.05.005

24. Kim BK, Yoo HI, Choi K, Yoon SK. miR-330-5p inhibits proliferation and migration of keratinocytes by targeting Pdia3 expression. FEBS J. 2015;282(24):4692-4702. doi:10.1111/febs.13523

25. Pierce AM, Schneider-Broussard R, Gimenez-Conti IB, Russell JL, Conti CJ, Johnson DG. E2F1 has both oncogenic and tumor-suppressive properties in a transgenic model. Mol Cell Biol. 1999;19(9):6408-6414. doi:10.1128/mcb.19.9.6408

26. Dapas B, Farra R, Grassi M, et al. Role of E2F1-cyclin E1cyclin E2 circuit in human coronary smooth muscle cell proliferation and therapeutic potential of its downregulation by siRNAs. Mol Med. 2009;15(9-10):297-306. doi:10.2119/ molmed.2009.00030

27. Stanelle J, Putzer BM. E2F1-induced apoptosis: turning killers into therapeutics. Trends Mol Med. 2006;12(4):177185. doi:10.1016/j.molmed.2006.02.002

28. Chen Q, Hung FC, Fromm L, Overbeek PA. Induction of cell cycle entry and cell death in postmitotic lens fiber cells by overexpression of E2F1 or E2F2. Invest Ophthalmol Vis Sci. 2000;41(13):4223-4231.

29. Guan A, Wang H, Li X, et al. MiR-330-3p inhibits gastric cancer progression through targeting MSI1. Am J Transl Res. 2016;8(11):4802-4811.

30. Alla V, Engelmann D, Niemetz A, et al. E2F1 in melanoma progression and metastasis. J Natl Cancer Inst. 2010;102(2):127-133. doi:10.1093/jnci/djp458

(c) 2019 The Author (s); This is an open-access article distributed under the terms of the Creative Commons Attribution License (http://creativecommons.org/licenses/by/4.0), which permits unrestricted use, distribution, and reproduction in any medium, provided the original work is properly cited. 